\title{
New resources to aid doctors in lowering opioid doses
}

\author{
口 Cite as: CMAJ 2019 July 29;191:E849-50. doi: 10.1503/cmaj.1095783
}

Posted on cmajnews.com on July 09, 2019.

$\mathbf{N}$ ew resources are emerging to help physicians across Canada reduce opioid doses for patients. They come at a time when doctors feel pressure from regulatory agencies to deprescribe opioids but don't feel trained or supported in the process.

"We spend a lot of time in medical schools teaching doctors how to start medications - not just opioids - and we don't really talk about stopping any of them," said Dr. Lori Montgomery, medical lead of the Calgary Pain Program for Alberta Health Services.

Montgomery developed the curriculum for a recently launched opioid deprescribing course, offered by the University of Calgary, with funding from Alberta Health Services and the College of Physicians and Surgeons of Alberta (CPSA).

The course covers the physiological response to tapering and how to motivate patients to taper, as well as how to schedule dosage reductions. It's delivered in a "flipped classroom" format, whereby physicians listen to podcasts and go through online modules before attending two classroom sessions, to be held this fall, to discuss the information as a group, said Dr. Kelly Burak, associate dean of continuing medical education at the University of Calgary.

The course is voluntary but the CPSA may require some doctors with large numbers of patients on high opioid doses to take it, Burak added.

"It's easy to start a prescription - you explain how to take it, you talk about the potential side effects, and the patient goes away," said Burak. "Stopping is really complex."

Montgomery stressed that patient buy-in is key. "If you force a patient to taper and they're not ready, then they

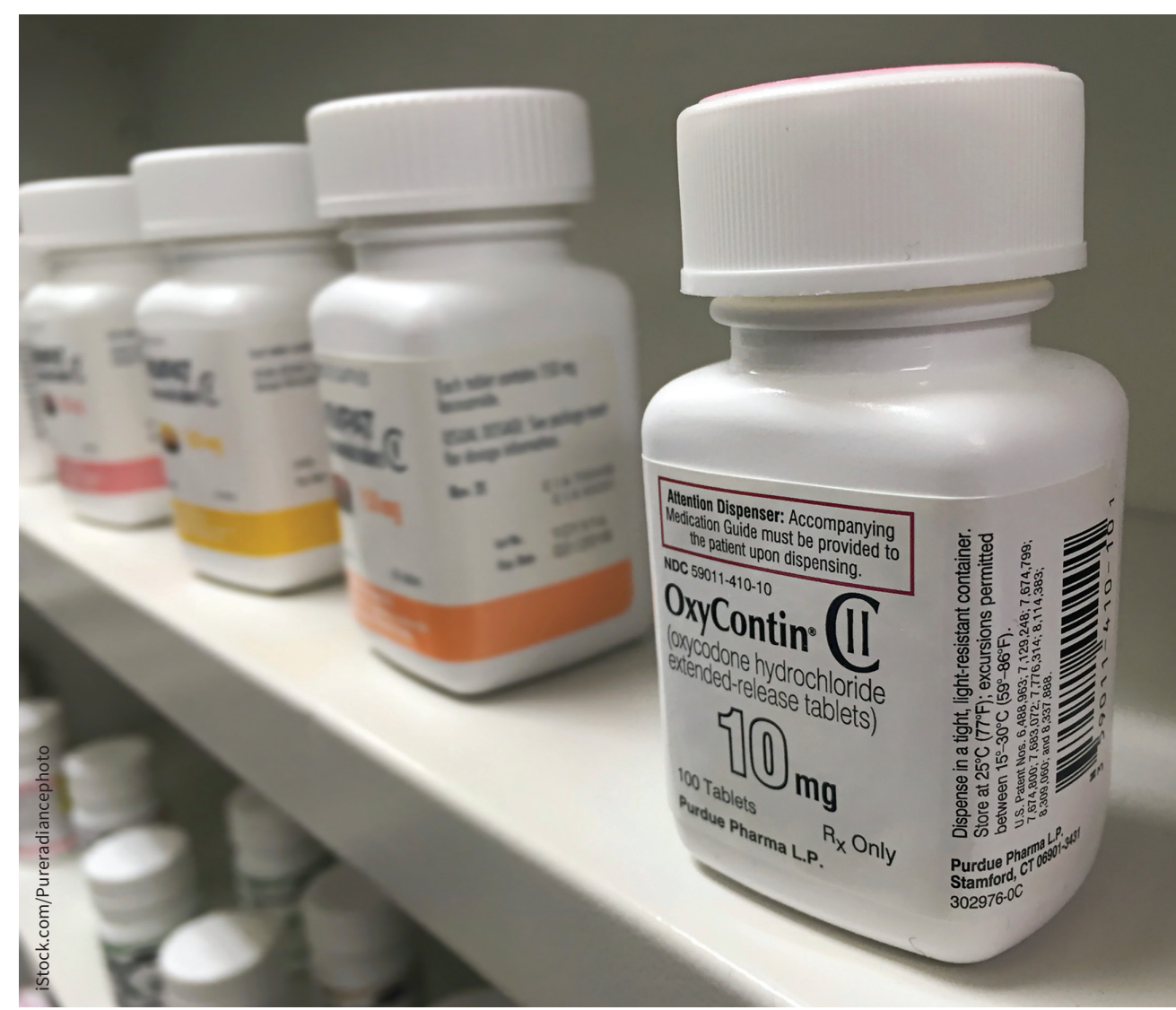

Physicians learn much more about starting patients on medications than tapering or taking patients off medications.

may seek out illicit opioids ... and there's a high chance the first time they do that it could be fatal."

Though most patients feel less pain and have a better quality of life after tapering opioids for non-cancer chronic pain, some may experience more psychological and physical pain if they feel anxiety about being forced into a course of action. Montgomery stressed that opioid reduction should never be pitched as "all or nothing," and patients should be reminded that the taper can be paused at any time.
Pitching a small reduction in opioid dosage as a "trial" for a patient can also be effective. "It can be really reassuring for the patient if they make a tiny dose reduction and they notice absolutely nothing," said Montgomery. If a patient has an opioid use disorder, however, a different course of action, often including opioid replacement therapy, may be required.

Physicians and pharmacists at the Canadian Deprescribing Network have launched resources for both prescribers 
and patients, including a video and educational brochures. Justin Turner, senior science advisor at the network, is leading a study to look at whether a mailed brochure could lead to a reduction in opioid prescriptions.

Previous research by the network found that $43 \%$ of patients inappropriately prescribed sedatives discontinued the medication after they and their doctors were targeted with education by community pharmacists, compared to $12 \%$ in the control group.

For both patients and prescribers, the Canadian Deprescribing Network has an online calculator "to take the guesswork and confusion" out of opioid tapering,
Turner explained. The user clicks on the opioid they're taking, enters their dosage and frequency, and the calculator provides a tapering schedule with images of the pills. The calculator rounds up to a whole pill because most opioids are tamper proof and can't be cut.

According to Turner, doctors can safely taper dosages by $10 \%$, reducing the dosage every one-to-four weeks, depending on the patient's preference. He recommends that doctors advise patients to keep pain diaries before tapering "so they're not looking back with rose coloured glasses" about their pain.

"There's a lot of data showing, on average, patients' pain does not get worse when they reduce their opioids and, on average, their quality of life gets better," he said.

Dr. Cara Tannenbaum, founder and cochair of the deprescribing network, points out that funding models continue to make the deprescribing process difficult for patients and their doctors, however. "We talk about pacing, cognitive behaviour therapy, physiotherapy, all of which has good evidence for chronic pain," she says, "but in many provinces, a psychologist isn't even covered and physiotherapy outside of the hospital isn't covered."

Wendy Glauser, Toronto, Ont. 